\title{
Improving sensitivity of a small angle x-ray scattering camera with pinhole collimation using separated optical elements
}

\author{
Th. Zemb, O. Taché, F. Né, and O. Spalla ${ }^{a}$ \\ Service de Chimie Moléculaire (D.R.E.C.A.M./C.E.A.), C.E./Saclay, F91191 Gif sur Yvette Cedex, France
}

(Received 12 July 2001; accepted 5 January 2003)

\begin{abstract}
We show that a significant improvement in the sensitivity of a Huxley-Holmes design for a small angle $\mathrm{x}$-ray scattering camera is obtained by separating the mirror and the monochromator. The design of the camera involves a long $\mathrm{x}$-ray mirror close to a point $\mathrm{x}$-ray source associated with a curved focusing crystal located close to the sample. The sample area is located at half the distance between the source and detector planes. Diffuse scattering produced by the mirror is not incident on the focusing crystal, thus reducing the background signal. Complete elimination of hard $\mathrm{x}$ rays allows precise calibration and hence absolute determination of sample cross section by means of a semitransparent beam stop. In pinhole geometry, the flux corresponds to a $\sim 10^{7}$ photons/s through the sample, collimated to $10^{-2} \AA^{-1}$ in $q$ range. This allows determination of scattered intensities on the order of $10^{-3} \mathrm{~cm}^{-1}$, corresponding to the scattering related to isothermal compressibility of less than $0.1 \mathrm{~mm}$ of pure water. As a reference sample, the widely used Lupolen ${ }^{\mathrm{TM}}$, a semicrystalline polymer, is calibrated. The high- $q$ limit $\left(q \approx 4.5 \mathrm{~nm}^{-1}\right)$ of a porous calcite sample can be used as a secondary standard for specific area determination of solid/solid or solid-liquid dispersions.

(C) 2003 American Institute of Physics. [DOI: 10.1063/1.1556954]
\end{abstract}

\section{INTRODUCTION}

According to the classical Huxley-Holmes design, ${ }^{1}$ a camera used for general experiments in small angle x-ray scattering (SAXS) should work in the so-called pinhole geometry. Using a mirror, a bent monochromator and a point source avoids the delicate problem of desmearing raw data in order to obtain the scattered intensity ${ }^{2} I(q)$. Pinhole geometry has allowed for instance the identification of the DNA double helix from oriented gels. ${ }^{3}$

Interaction peaks in colloidal samples made of repulsive particles such as micelles are difficult to measure without a pinhole geometry. ${ }^{4}$ Our aim is to increase the sensitivity of a pinhole camera, in order to be able to analyze a wide range of different samples, including anisotropic and very low contrast, by improving signal over background ratio and avoiding desmearing procedures. This requires the ratio between the background count rate at the pixel corresponding to the lowest scattering angle recorded and the number of photons passing through the sample (defining the so-called limiting factor) to be less than $10^{-8}$. In our setup, these low-angle pixels correspond to Bragg spacing on the order of $60 \mathrm{~nm}$. Our goal is to realize on a laboratory source:

typical counting times for weakly scattering samples such as pure water or small ionic micelles on the order of $1 \mathrm{~h}$, and a few minutes for inorganic colloids;

(ii) the advantage of obtaining directly absolute intensity without desmearing; and

(iii) avoiding the necessity of a secondary standard.

\footnotetext{
a) Author to whom correspondence should be addressed; electronic mail: spalla@drecam.saclay.cea.fr
}

\section{DESIGN OF THE CAMERA}

\section{A. General layout of the camera}

The general layout of the camera is shown in Fig. 1. A rotating copper anode provides a point source $(50 \mathrm{kV}, 300$ $\mathrm{mA})$. This source is used with the classical takeoff angle of $6^{\circ}$. Thus, the apparent size of the source is $1 \mathrm{~mm} \times 1 \mathrm{~mm}$ full width half maximum (FWHM). The horizontal $\mathrm{x}$-ray beam penetrates through a beryllium window in a vacuum chamber, through a set of removable calibrated attenuators and two different optical devices, a planar x-ray mirror, and a bent germanium crystal as monochromator. After the sample area, where thermostated rotating sample holders or chambers for control of the osmotic pressure may be inserted, ${ }^{5}$ the $\mathrm{x}$-ray beam enters into a second conic vacuum chamber. The input window of this chamber is made of a $50 \mu \mathrm{m} \mathrm{Littrex}^{\mathrm{TM}}$ producing negligible parasitic scattering as compared to Kapton $^{\mathrm{TM}}$ and Mylar ${ }^{\mathrm{TM}}$ (see Fig. 2). The $300 \mathrm{~mm}$ diam circular output window is a carbon-epoxy fiber plate of thickness $0.3 \mathrm{~mm}$. The scattering is recorded with a sample to detector distance of $2160 \mathrm{~mm}$. The direct beam hits a semitransparent beam stop in a position as asymmetric as possible in order to increase the spatial frequency dynamic range $\left(q_{\max } / q_{\min }=30\right)$. An image plate used as a detector when resolving Bragg peaks is crucial or scattered intensity close to the beam stop is required precisely. Otherwise, two dimensional gas position sensitive detectors of the Gabriel type ${ }^{6}$ are used routinely. Limitations in local dynamic range, amplification stability, and resolution due to avalanche shape have been reviewed by Petrascu et $_{\text {al. }}{ }^{7}$ 


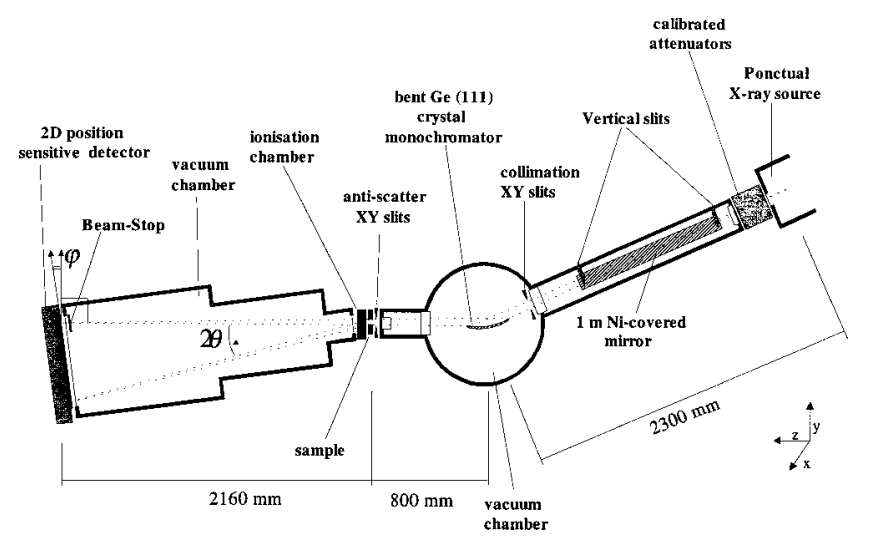

FIG. 1. General layout of the separated optics camera. The horizontal mirror under grazing incidence is used to filter out hard $\mathrm{x}$ rays and is located as close as possible from the $\mathrm{x}$-ray source, in order to prevent nonspecular reflection from arriving on the focusing monochromator. $2 \theta$ is the scattering angle, $\varphi$ is the small angle between detection plane, and the normal to scattered beam.

\section{B. Optical elements}

\section{X-ray mirror}

The $\mathrm{x}$-ray mirror is a $1 \mathrm{~m}$ long piece of float glass coated by a $50 \mu \mathrm{m}$ chemical vapor deposition nickel layer. This efficient $\mathrm{x}$-ray mirror, which absorbs the radiation harder than $\mathrm{Cu} K_{\alpha}$ at grazing incidence, is located as close as possible to the x-ray source. For the $\mathrm{Cu} K_{\alpha}$ radiation, the critical reflection angle is $5.5 \mathrm{mrad}$. Optimization of the limiting factor and hence the sensitivity requires an average incidence angle of 4 mrad. ${ }^{8}$ The horizontal level of the mirror and its angle with the horizontal (4 mrad) is adjusted with the help of a laser in order to produce a horizontal reflected $\mathrm{x}$-ray beam. For hard $\mathrm{x}$ rays of $\lambda_{\mathrm{Cu}} / 3$, which would pass through the monochromator, the incidence angle on the mirror is nearly three times the critical reflection angle. Two horizontal slits avoid direct illumination of the monochromator by the source. Therefore, this particular setting of this camera

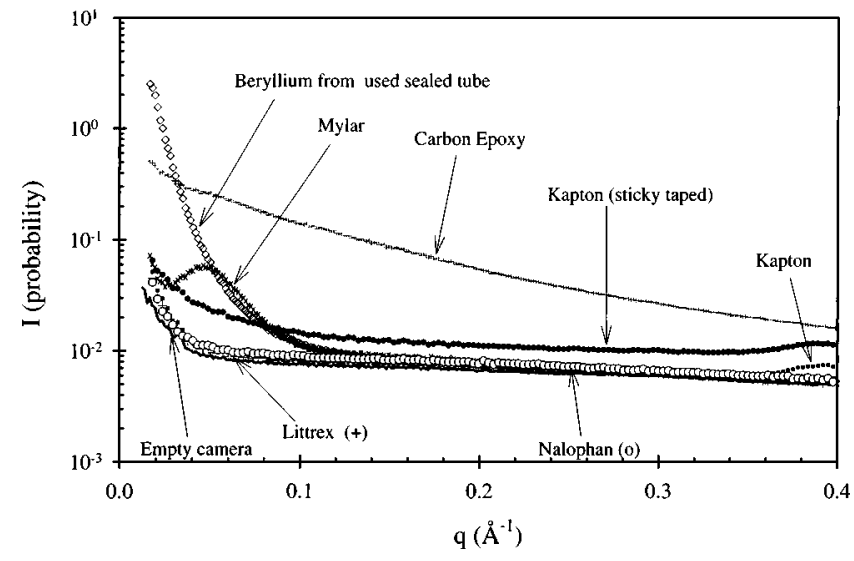

FIG. 2. Scattering cross section of windows, measured in sample position, compared to the scattering obtained with the empty camera: Littrex (transmission $T=0.98$, thickness $t=50 \mu \mathrm{m}$ ), carbon-epoxy fiber plate (transmission $T=0.75$, thickness $t=350 \mu \mathrm{m}$ ), Mylar (transmission $T=0.91$, thickness $t=50 \mu \mathrm{m}$ ), Kapton windows (transmission $T=0.98$, thickness $t$ $=30 \mu \mathrm{m}$ ) and Nalophan (transmission $T=0.98$, thickness $t=50 \mu \mathrm{m}$ ) compared to the scattering of the empty camera; units is scattering probability, i.e., absolute scattering for a length unit sample thickness. avoids beam-hardening through the attenuator, hence allowing measurement of sample transmission coefficient for a pure $\mathrm{Cu} K_{\alpha}$ beam.

\section{Bent monochromator}

In order to further filter out hard x rays as well as the $K_{\beta}$ radiation produced by the source, the camera is equipped with a bent germanium crystal, cut at $\alpha=2^{\circ}$ to the (111) crystallographic plane, and with a radius of curvature of 11.5 $\mathrm{m}$ optimized to let the detector plane be the focal plane. This crystal is roughly located in the middle of the camera, at $\mathrm{SC}=2.30 \mathrm{~m}$ from the source and at $\mathrm{CF}=2.94 \mathrm{~m}$ of the detector plane. The focusing condition was first given by Johann ${ }^{9}$

$$
\mathrm{SC}=\mathrm{CF} \frac{\sin \left(\theta_{B}-\alpha\right)}{\sin \left(\theta_{B}+\alpha\right)}=\mathrm{CF} \cdot b,
$$

where $b$ is the asymmetry factor.

For germanium reflecting copper $K_{\alpha}$ radiation, the Bragg angle $\theta_{B}$ is $13.21^{\circ}$. The asymmetric ratio induces a slight magnification of the source in the detector plane SC/CF $=0.78$. Due to the large acceptance angle of asymmetrically cut bent germanium, the whole length of the monochromator is then efficient for focusing ${ }^{10}$

$$
\omega_{\mathrm{acc}}=\omega_{0} / \sqrt{b} \text {. }
$$

In the case of the separated optics camera based on a germanium crystal, the acceptance angle is $90 \mu \mathrm{rad}$ and the divergence angle is $70 \mu \mathrm{rad}$, since $\omega_{0}$ is $80 \mu \mathrm{rad}$ for the quality of germanium used. ${ }^{11}$ This natural divergence of an asymmetric Ge crystal sets the minimum $q$ accessible for measurements. To optimize the flux through the sample, the whole source must be seen with angles less than the acceptance angle from any point of the monochromator. Also, the beam-stop size must be contained within the divergence angle, as seen from any point of the monochromator. These conditions impose a total length of more than $4 \mathrm{~m}$ for the whole camera as well as a size of the beam stop, larger than the geometrical extension of the image of the source in the plane of the detector.

The monochromator is first optically adjusted using the procedure described by Spencer. ${ }^{12}$ A specially designed mirror bender (ACTAL, Canberra) imposes asymmetric couples on both ends of the thin rectangular Ge plate. Optimal thickness was found to be $0.25 \mathrm{~mm}$ : finer plates are too difficult to machine and thicker plates break when bent. To improve sensitivity of the camera, lateral "tails" observed near the edge of the beam stop have to be reduced. This strong parasitic scattering is observed when monochromators are used with the surface state after cut and flattening. The origin of this strong "tail" close to the beam stop is the residual surface roughness and defects introduced during cutting. At least 1 order of magnitude of reduction can be obtained by chemical etching, which has the advantage of removing the perturbed layer without introducing strain. Optimization of the etching procedure has to be performed. It has been found $^{13,14}$ that a few minutes of etching in warm acid mixture removes $40 \mu \mathrm{m}$ of surface and reduces the observed limiting factor in the horizontal plane by a factor of 10 . The 
quality of etching also controls the ratio of the beam-size FWHM in the detector plane to the beam-stop size. In the horizontal direction, the surface scattering of the crystal produces off-specular, i.e., nonfocusable reflection, which produces the residual scattering at the edge of the beam stop. ${ }^{11,14}$

\section{Slits}

The sensitivity of the separated optics camera is due to two pairs of $X-Y$ slits. The first one is located as close as possible to the exit of the $\mathrm{x}$-ray mirror. The guard slitssometimes called "antiscatter" slits—are located just in front of the sample area. Since the edges of the slits always produce some parasitic scattering, the limiting factor is inversely proportional to the square of the distance between extreme slits. The design of the camera allows an unusually large separation distance of $D=100 \mathrm{~cm}$ between the first and the guard slits. Therefore, the diffuse scattering produced by the mirror imperfections is not incident on the monochromator. Therefore, the beam emerging from the monochromator is free of hard x rays, as well as $\mathrm{Cu} K_{\beta}$ radiation, which is eliminated by the nickel layer, used under grazing incidence. The horizontal metallic edges limiting the beam to an adjustable width over the mirror (typically $3 \mathrm{~mm}$ ) also avoid scattering by the edges of the mirror.

Horizontal and vertical collimation slits ${ }^{15}$ are located between the mirror and the crystal (see Fig. 1). The quality of those slits is very important, since the parasitic scattering of those slits are incoming on the guard slits and thus play a major role in the sensitivity of the camera. The guard slits are a crossed pair of crossed slits with steel edges ${ }^{16}$ located as close as possible of the sample. The main slits are adjusted using an image plate imprint in order to use most of the available area of the monochromator which maintains the required limiting factor.

The guard slits are set in order to minimize the value of parasitic counting at the beam-stop edge relative to the total flux. When all slits are set, we can quantify the final sensitivity by defining and routinely measuring the so-called limiting factor $l_{f}$ :

$$
l_{f}=\frac{C_{\mathrm{vh}} / \Delta t}{\phi S},
$$

where $C_{\mathrm{vh}}$ is the number of photons detected during $\Delta t$ on the most intense pixel at the edge of the beam stop over the flux of photons incoming the sample area in the direct beam $(\phi \cdot S)$. The limiting factor is the ratio between the background count rate and the number of photons passing through the sample. After optimization of the slits, the weakest scattering samples should produce at least as much scattering as this background scattering (BS) produced by the empty camera at the edge of the beam stop in order to ensure safe background subtraction procedures. Limiting factors obtained in our setup are: $6.5 \times 10^{-9}$ top of the BS, 6.5 $\times 10^{-9}$ bottom of the BS, $28 \times 10^{-9}$ right side of the BS, and $14 \times 10^{-9}$ left side of the BS.
TABLE I. Comparison of the detector systems used.

\begin{tabular}{lll}
\hline \hline & Gas detection & Image plate \\
\hline Pixel size $(\mathrm{mm})$ & 0.7 & 0.088 \\
counts/pixel/s without $\mathrm{x}$ rays & $3.1 \times 10^{-4}$ & $5.5 \times 10^{-5}$ \\
counts $/ \mathrm{mm}^{2} / \mathrm{s}$ & $6.3 \times 10^{-4}$ & $7.1 \times 10^{-3}$ \\
Dynamic range & 3.5 decades & 5 decades \\
\hline \hline
\end{tabular}

\section{Beam stop}

We use a thin slab of nickel of thickness $300 \mu \mathrm{m}$ (attenuation factor 204000 ) cut at a size of $21 \mathrm{~mm} \times 42 \mathrm{~mm}$ in order to attenuate the direct beam by roughly $53 \mathrm{db}$. The beam stop is made of nickel foil of thickness $t=0.30 \mathrm{~mm}$. The expected attenuation is estimated from the mass attenuation factor $\mu=45.7 \mathrm{~cm}^{2} / \mathrm{g}\left(\mathrm{Cu} K_{\alpha}\right)$ and density $\rho=8.9$ $\mathrm{g} / \mathrm{cm}^{3}$ :

$$
A=\frac{J_{\text {out }}}{J_{\text {in }}}=e^{-\mu \rho t} .
$$

The expected attenuation is $A=2.10^{5}$.

The protocol to measure the beam-stop attenuation is the following. We use a second attenuator of predetermined attenuation factor of 500 and make exposures of the direct beam using the 5 decades of linearity in the signal/dose ratio of the image plate readout system, typically $1 \mathrm{~min}$ without beam stop and 1 night with beam stop. The ratio of integrated value of the small spot left by the direct beam gives the beam-stop attenuation factor. The experimental value is $A=204000$ with a few percent reproducibility.

Finally, the count rate due to scattering of the sample near the beam stop and the image of the direct beam through the sample are of the same order of magnitude, thus allowing dead-time corrections to be neglected.

\section{Position sensitive systems}

To record SAXS patterns, we use either a large twodimensional (2D) position sensitive gas chamber, built by Gabriel $^{6}$ (EMBL outstation, Grenoble) or an image plate reader. ${ }^{17}$ The gas chamber is a $2 \mathrm{D}$ multiwire gas position sensitive detector $512 \times 512$ pixels, filled with 1.6 bar xenon/ ethane mixture. The largest available gas chamber has an effective diameter of $30 \mathrm{~cm}$, with a carbon sheet as an entry window, and an electronic resolution of $0.9 \mathrm{~mm}$. With a 3 $\mathrm{mm}$ pitch and low resolution, the dark count rate per pixel is typically reduced to $3.1 \times 10^{-4}$ counts per pixel and per second. Possible artifacts in measurements introduced by nonideal counting of gas position sensitive detectors have been discussed in detail previously. ${ }^{18}$

Using the image plates as well as the gas detectors and associated electronics, an adsorbed radiation dose of 1 photon/pixel produces well identified signals in the readout system for both technologies. The ratio of pixel area for the two systems is 65 (see Table I) and therefore gives the ratio of resolution as well as the ratio in sensitivity. Cosmic background can be electronically discriminated with the gas detector, but not with the image plate, since it is an integrating device. The dynamic range of typical image-plate based sys- 
TABLE II. Characteristics of the camera.

\begin{tabular}{|c|c|}
\hline Flux through the sample & $2 \times 10^{7}$ photons $/ \mathrm{s}$ \\
\hline Vertical collimation (mrad) & $0.5 \mathrm{mrad}$ \\
\hline Horizontal collimation (mrad) & $0.07 \mathrm{mrad}$ \\
\hline Brilliance (photons/s mrad ${ }^{2}$ & $10^{9}$ \\
\hline \multicolumn{2}{|l|}{$0.1 \%$ bw) } \\
\hline Beam size at sample (FWHM) & $2 \times 2 \mathrm{~mm}$ \\
\hline Beam-stop size & $21 \times 42 \mathrm{~mm}$ \\
\hline \multirow[t]{2}{*}{ Dynamic range $q_{\max } / q_{\min }$} & Gas detector $30 ; 0.015-0.45 \AA^{-1}$ \\
\hline & Image plate $60 ; 0.01-0.7 \AA^{-1}$ \\
\hline \multirow{4}{*}{$\begin{array}{l}\text { Background in the middle } \\
\text { of } q \text { range with } \mathrm{x} \\
\text { rays }\end{array}$} & $\begin{array}{l}7.3 \times 10^{-3} \text { counts/pixel/s for gas } \\
\text { detector }\end{array}$ \\
\hline & $1.5 \times 10^{-2}$ counts $/ \mathrm{mm}^{2} / \mathrm{s}$ \\
\hline & $\begin{array}{l}1.8 \times 10^{-4} \mathrm{counts} / \mathrm{pixel} / \mathrm{s} \text { for image } \\
\text { plate }\end{array}$ \\
\hline & $2.3 \times 10^{-2}$ counts $/ \mathrm{mm}^{2} / \mathrm{s}$ \\
\hline
\end{tabular}

Photons detected with empty camera (no sample in place) in the whole $2 \mathrm{D}$ data set

500 counts/s for gas detector

220 counts/s for image plate

tems, as integrating devices, rely on the linearity of a light intensity to electric charge conversion, which is currently limited to 5 decades if cascades of logarithmic amplifiers are used. ${ }^{17}$ Detectors based on ionization of gas cannot usually extend the dynamic range above 3 decades due to diffusion without ionization within the gas, as described in detail in Ref. 17.

Scattering cross sections, i.e., absolute intensity multiplied by the respective thickness of the parasitic scattering introduced by the presence of windows in the setup, are shown in Fig. 2, and compared to the total scattering of the empty camera.

After adjustment, the technical characteristics of the camera are summarized in Table II for $15 \mathrm{~kW}$ source electrical power. At this power, the total brilliance of the source is on the order of $10^{9}$ photons $/ \mathrm{s} / \mathrm{mm}^{2} / 0.1 \%$ bandwidth. The total entry angle used by the input slits of the camera is 3.5 $\times 10^{-2} \mathrm{mrad}^{2}$. The theoretical flux is therefore almost comparable to the maximum possible brilliance ${ }^{19}\left(3 \times 10^{7}\right)$. The difference is due to loss in windows.

\section{ABSOLUTE SCALING}

\section{A. Calibration of the $Q$ range}

Liquid crystals of the lyotropic type, cubic, hexagonal phases, as well as colloidal crystals exhibit long range order and sharp Bragg peaks. The value of the scattering angle at the maximum of the first order needs to be measured with precision, while the higher orders, usually weaker, are observed if the sensitivity of the camera is sufficient. $q$ in reciprocal length is given by

$$
q=\frac{4 \pi}{\lambda} \sin (\theta) \quad \text { with } \quad \tan (2 \theta)=\frac{d}{D},
$$

where $\lambda$ is the incident wavelength and $\theta$ is half of the scat-
TABLE III. Values of $q\left(\AA^{-1}\right)$ of Bragg peaks in the SAXS domain from dry alcohol crystals in powder average used for detector calibration.

\begin{tabular}{cc}
\hline \hline Tetradecanol & Octadecanol \\
\hline 0.1583 & 0.1525 \\
0.3167 & 0.3050 \\
0.4749 & $\cdots$ \\
0.6334 & $\cdots$ \\
\hline \hline
\end{tabular}

tering angle, which can be evaluated for each pixel knowing the distance $d$ to the center of the direct beam in the detector plane, located at distance $D$ to the sample.

The position of the Bragg peaks, after radial averaging, may be routinely checked by the use of a secondary standard. Long chain fatty alcohol may be used to check calibration, as suggested by Nakamura et al. ${ }^{20}$

Exact value of the spacing can only be determined if the exact location of the detection plane in the gas detector is determined. In order to determine the effective sample to detection plane distance, we use dry octadecanol, showing sharp Bragg peaks indicated in Table III. We use the two types of detectors for specific purposes. The resolution of the solid state detector (image plate with $88 \mu \mathrm{m}$ pixel) is higher than that of the gas detector but it requires roughly four times more accumulation time as shown in Fig. 3. Thus, when high resolution around the Bragg peak, for instance, is required the image plate will be used (with a section along the horizontal axis as the beam is focused in that direction). On the other hand, when good statistics on the absolute scaled intensity is required on a radially average spectrum, the gas detector is preferred.

\section{B. Absolute intensity}

The scaled scattered intensity produced by the sample is evaluated using the relation

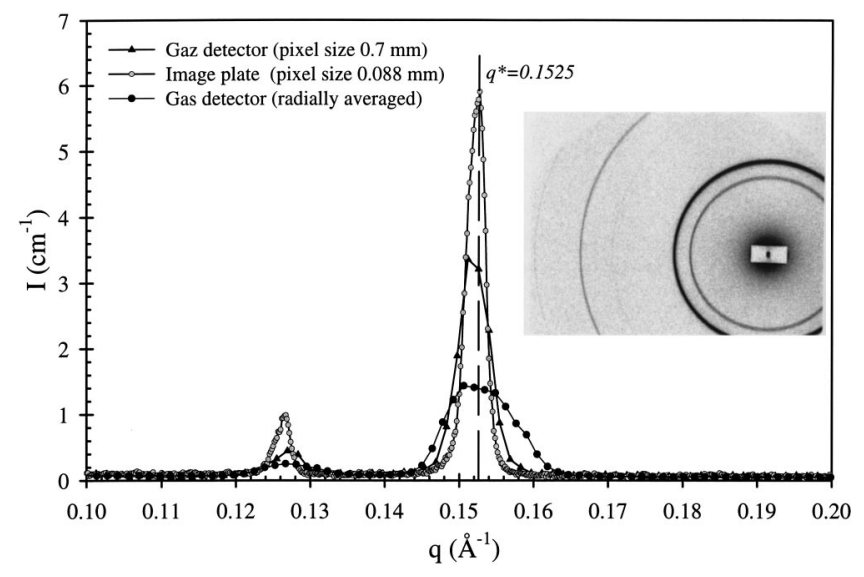

FIG. 3. (Insert) Readout obtained from an image plate exposed $4 \mathrm{~h}$ to the scattering of octadecanol. (Main) One-dimensional section (average on a few lines) is shown together with the same section on a gas detector ( $1 \mathrm{~h}$ of exposure time) image and the radially averaged spectrum obtained with the gas detector. Radial averaging damages the final resolution, due to convolution by the image of the source. On a vertical cut where resolution is best, gas and image plate detection can be compared, balancing sensitivity, which is better with a gas detector vs resolution, which is better with an image plate. The radially averaged gas detector signal is broadened due to the slightly larger dimension of the image of the source in the detector plane. 


$$
I(q)=\frac{1}{V} \frac{d \sigma}{d \Omega}=\frac{\eta_{1} C_{i j}}{\eta_{2}\left(\phi_{0} S T\right) d t} \frac{1}{\Delta \Omega} \frac{1}{e},
$$

where the scattered intensity $I(q)$ is defined by the differential scattering cross-section density per unit volume of sample; $C_{i j}$ is the number of counts detected on pixel $i j$ during $d t ; \eta_{2}$ is the detector quantum efficiency for the counts $C_{i j} ; \eta_{1}$ is the detector quantum efficiency when measuring the direct beam; $\left(\phi_{0} S T\right)$ is the flux (in detector units: counts/s) integrated over the whole beam transmitted by the sample; $T$ is the transmission of the sample; $\Delta \Omega$ is the solid angle covered by 1 pixel seen from the center of the sample: $\Delta \Omega=p^{2} / D^{2}$, where $p$ is the pixel size and $D$ the sample to detector distance; and $e$ is the thickness of the sample $(\mathrm{cm})$.

For absolute scaled measurement, one has to ensure that $\eta_{1}=\eta_{2}$. As already mentioned, we use a semitransparent beam stop of transmission typically 1/204000 in order to record the direct beam through the sample and the small angle scattering signal of roughly the same intensity. Then, we can use the product $\left(\phi_{0} \cdot S \cdot T \cdot d t\right) \cdot T_{\mathrm{BS}}$ as a monitor which can be obtained by integrating the direct beam during $d t$ through the semitransparent beam stop of previously measured transmission coefficient $T_{\mathrm{BS}}$. This procedure is reliable as long as there is a negligible hard $\mathrm{x}$-ray component in the direct beam.

\section{Determination of the transmission of the sample and beam stability monitoring}

In order to ensure reliable empty cell subtraction, and thus good reproducibility of calibration using the scattering of pure water, the transmission of the sample has to be measured with good accuracy.

The transmission is measured using an ionization chamber located after the sample. The device consists of two conducting plates, each with an area of $\sim 5 \mathrm{~cm}^{2}$, separated by 2 $\mathrm{cm}$. A homogeneous electric field of $100 \mathrm{~V} / \mathrm{cm}$ is generated between the plates. For a flux on the order of $10^{6}$ photons $/ \mathrm{s}$ in the beam of size $3 \times 2 \mathrm{~mm}$ at the position of the ionization chamber, an induced current of typically $200 \mathrm{pA}$ is measured using a conventional picoammeter. The reproducibility and reliability of this simple device is ensured using ultrathin Mylar windows which isolate air inside the ionization chamber from the laboratory atmosphere. Moisture is avoided by inserting a small quantity of a dessicating agent into the ionization chamber. The transmission of the sample is measured by the ratio of current measured with and without the sample, which is located a few centimeters before the ionization chamber.

Once the absolute intensities are radially averaged, scattering due to a sample can be obtained by direct subtraction of the background

$$
I(q)=\frac{1}{\Delta \Omega}\left[\left(\frac{C_{i j}}{\left(\phi_{0} S d t\right) T}\right)-\left(\frac{C_{i j}^{e c}}{\left(\phi_{0} S d t\right) T_{e c}}\right)\right] \frac{1}{e},
$$

where the background, $C_{i j}^{e c}$, is taken with an empty cell in place (measurement of liquids), or a cell filled with pure solvent (case of diluted colloids).

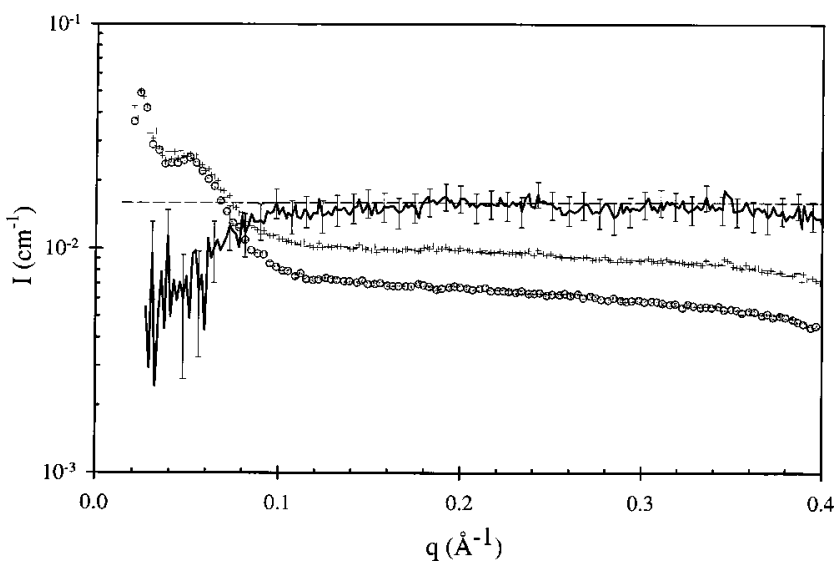

FIG. 4. Absolute intensity obtained for a water sample of transmission $T$ $=0.19$ and for an accumulation time of $4 \mathrm{~h}$ (mylar windows thickness 50 $\mu \mathrm{m}$ of transmission 0.98 each). $(\bigcirc)$ is the normalized scattering with an empty sample cell $(+)$ is the scattering with a $1.5 \mathrm{~mm}$ sample of water. Dots and error bars are the result of subtraction, as compared to the expected value of the scattering calculated from compressibility (dashed line).

\section{Calibration of absolute scaling of SAXS experiments using water}

Pure solvents can be used as secondary standards to calibrate the whole setup. ${ }^{21}$ Nevertheless, our setup does not require a secondary standard and we can indeed measure the absolute scattering of water and other solvents. As an example, we compare below the theoretical value for water to the direct absolute value that can be measured with the setup. The low- $q$ limit of small angle scattering arising from a compressible pure solvent is given by ${ }^{22}$

$$
I(q=0)=\left(\rho f_{e}^{2} n_{e}^{2}\right) \rho k T \chi_{T},
$$

where the leading terms in brackets represent the scattering of independent molecules of density $\rho_{w}$ and multiplied by the isothermal compressibility (the constant $f_{e}=0.282$ $\times 10^{-12} \mathrm{~cm}$ is the electron "Thomson scattering length"). Pure water has a density of $\rho=3.3 \times 10^{22}$ molecules $/ \mathrm{cm}^{3}$, the number of electrons per water molecule is 10 , and the isothermal compressibility is $\chi_{t}=45.7 \times 10^{-11} \mathrm{~Pa}^{-1}(298 \mathrm{~K})$. Finally, the scattering produced by pure water used as a reference standard is $I_{\text {water }}(q=0)=1.62 \times 10^{-2} \mathrm{~cm}^{-1}$.

It should be noted that careful control of temperature is important in calibration experiments, since the compressibility of water varies with temperature. ${ }^{23}$ The raw data obtained on our setup with a $1.5 \mathrm{~mm}$ thick sample between Mylar windows are shown in Fig. 4. The experimental value that is found is in good agreement with the calculated value. This value was confirmed experimentally by Hendricks. ${ }^{24}$

Other solvents used as an internal standard in samples or for calibration are

$$
\text { - Dodecane } \quad \chi_{t}=98.7 \times 10^{-11} \mathrm{~Pa}^{-1}
$$

and

$$
\begin{aligned}
& I_{\text {dodecane }}(q=0)=2.15 \times 10^{-2} \mathrm{~cm}^{-1}, \\
& \text { - Hexane } \quad \chi_{t}=16.5 \times 10^{-10} \mathrm{~Pa}^{-1}
\end{aligned}
$$

and

$$
I_{\text {hexane }}(q=0)=2.87 \times 10^{-2} \mathrm{~cm}^{-1} .
$$




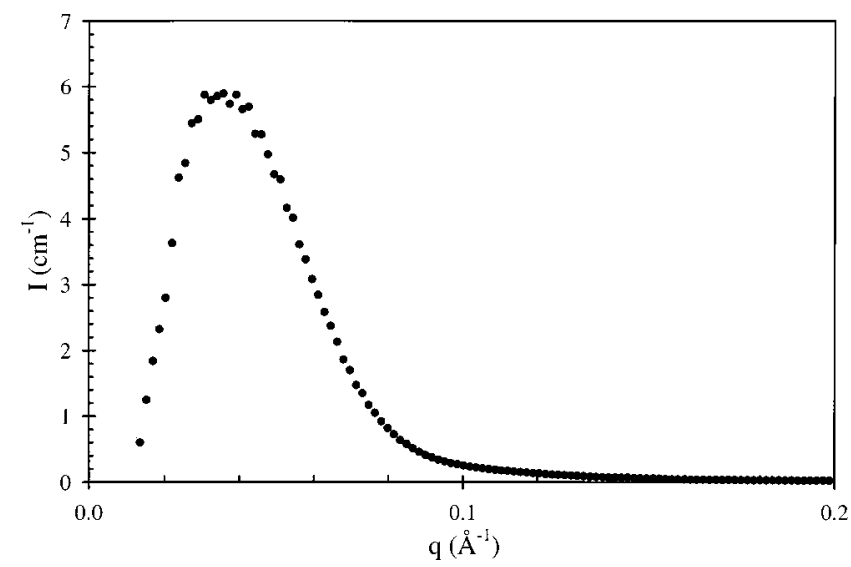

FIG. 5. Scaled SAXS intensity obtained for the Lupolen ${ }^{\mathrm{TM}}$ sample of thickness $t=3 \mathrm{~mm}$ and transmission $T=0.37$ (exposure time $15 \mathrm{mn}$ ).

\section{Measuring strong secondary reference scatterers such as Lupolen ${ }^{T M}$}

Lupolen is a semicrystalline high molecular weight polyethylene produced by BASF. This sample produces a very strong signal, nearly 3 orders of magnitude stronger than pure water. At $q=0.03 \AA^{-1}$, a scattering maximum is observed. This strong signal with a broad peak at a convenient angle can also be easily desmeared when using a slit geometry. It is a solid, so no windows are required and it is not subject to aging. For these reasons, the scattering of Lupolen is widely used as a secondary reference standard. Wignall ${ }^{25}$ has determined the differential cross section per unit length for this sample with different geometries. This sample, used in a large number of laboratories as a reference, has a scattered intensity peak of $6 \pm 0.1 \mathrm{~cm}^{-1}$ at $q=0.03 \AA^{-1}$. The result obtained in the separated optics camera is shown in Fig. 5. Strong signals such as those produced by solid polymers are easily measured within 15 min with good statistics.

\section{Using the high sensitivity of the separated optics camera for strongly decreasing signals}

Porous or biphasic heterogeneous samples produce a characteristic signal decaying sharply with $q$. Conditions to obtain a pure "Porod decay" 26 when the intensity at large $q$ asymptotic limit decays as $q^{-4}$, taken between limits $q_{\text {inf }}$ and $q_{\text {sup }}$, are as follows: ${ }^{27}$

(i) The interface is "sharp", i.e., the transition from media 1 to media 2 occurs in a distance much smaller than $2 \pi / q_{\text {Max }}$, so that fluctuation of the electronic density inside both media can be neglected. Average "particle" diameter or grain size, interparticle distance, and inverse of average curvature of interface are all at least 5-10 times larger than $1 / q_{\min }$.

(ii) Structural polydispersity is large enough to dampen the oscillations in scattering form factor.

Under these conditions, the Porod decay is given by

$$
\lim \left(I(q) q^{4}\right)_{q \rightarrow \infty}=2 \pi \Sigma(\Delta n)^{2},
$$

where $(\Delta n)^{2}$ is the square of the scattering length density difference $\left(\mathrm{cm}^{-2}\right)$, and the specific area per unit volume $\Sigma$

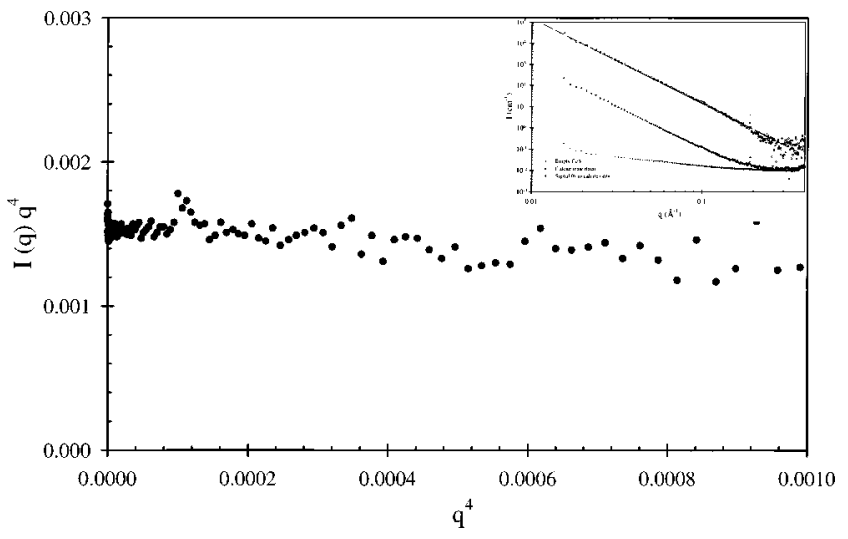

FIG. 6. Scattering of samples producing steeply decreasing power-law behavior. (Inset) Result shown in log-log scale for the dry solid. (Main) Porod representation $I(q) \cdot q^{4}$ function of $q^{4}$ produced by calcite powder used for calibration. This representation allows determination of Porod constant independently from value of flat parasitic background. Taking $\Delta n$ $=0.815 \mathrm{e} / \AA^{3}$ as the contrast between air and calcite, the specific area determined from Porod plot as well as BET is $17 \mathrm{~m}^{2} / \mathrm{g}$ as compared to the 20 $\mathrm{m}^{2} / \mathrm{g}$ nominal value (transmission 0.28 , average powder thickness $65 \mu \mathrm{m}$ ) of the calibration sample supplied and cross-checked by P. Delord (Montpellier University).

$\left(\mathrm{cm}^{2} / \mathrm{cm}^{3}\right)$ contains "open" as well as inaccessible pores. For granulometry of submicron size, the high- $q$ Porod regime corresponds to the small angle part of the SAXS intensity, thus allowing a nondestructive determination of surface-tovolume ratio in emulsions. ${ }^{28}$ The advantage of direct structural determination by SAXS over the more widespread BET method is that inaccessible closed volumes contribute to the Porod decay behavior. As an example, the specific surface of a widely used industrial calcite powder (Socal 30, produced by the Solvay Company) extracted from the Porod limit is shown in Fig. 6. The experimental value, $17 \mathrm{~m}^{2} / \mathrm{g}$ is in very good agreement with the Brunauer-Emmett-Teller determination made by P. Delord (Montpellier University).

\section{ACKNOWLEDGMENTS}

The authors acknowledge Jacques Lambard for the mechanical design of the camera and Didier Gazeau, who was responsible for the camera while in our laboratory, for valuable discussions, and the development of calibration procedures.

${ }^{1}$ For a review of classical SAXS designs, see H. E. Huxley and W. Brown, J. Mol. Biol. 30, 383 (1967).

${ }^{2}$ O. Yoda, J. Appl. Crystallogr. 17, 337 (1984).

${ }^{3}$ For a discussion of this point, see G. S. Stent in a critical edition of The Double Helix, edited by J. D. Watson (W. W. Norton, Norton, New York, 1980).

${ }^{4}$ K. Müller, in Small Angle X-ray Scattering, edited by O. Glatter and O. Kratky (Academic, London, 1982).

${ }^{5}$ M. Dubois and Th. Zemb, J. Phys. IV 8, 55 (1998).

${ }^{6}$ A. Gabriel, Rev. Sci. Instrum. 48, 1303 (1977).

${ }^{7}$ A. M. Petrascu, M. H. J. Koch, and A. Gabriel, J. Macromol. Sci., Phys. 37, 463 (1998).

${ }^{8}$ E. Erola, V. Etelaniemi, P. Suortti, Ph. Pattison, and W. Thomson, J. Appl. Crystallogr. 23, 35 (1990).

${ }^{9}$ A. Guinier and G. Fournet, Small Angle Scattering of X-Rays (Wiley, New York, 1995), Chap. 3.

${ }^{10}$ T. Matsushita and U. Kaminaga, in Handbook on Synchrotron Radiation, edited by E. Koch (Elsevier, Amsterdam, 1983), Vol. 1, p. 261. 
${ }^{11}$ J. Lambard, P. Lesieur, and Th. Zemb, J. Phys. I 2, 1191 (1992).

${ }^{12}$ M. J. Spencer, J. Appl. Crystallogr. 11, 715 (1978).

${ }^{13}$ H. Shiwaku and H. Hyodo, Jpn. J. Appl. Phys., Part 2 30, L2065 (1991).

${ }^{14}$ M. Amagalian, D. K. Christen, A. R. Drews, C. J. Clinkak, H. Matsuoka, and G. G. Wignall, J. Appl. Crystallogr. 31, 235 (1998).

${ }^{15}$ Sietronics, Canberra, Producing slits with Tantalum edges, Australia.

${ }^{16}$ Slits with crossed stainless steel edges, Huber, Germany, ref. 3013/1.

${ }^{17}$ F. Né, D. Gazeau, J. Lambard, P. Lesieur, Th. Zemb, and A. Gabriel, J. Appl. Crystallogr. 26, 763 (1993).

${ }^{18}$ F. Né, A. Gabriel, M. Koksis, and Th. Zemb, J. Appl. Crystallogr. 30, 306 (1997).

${ }^{19}$ V. Honkimäki, J. Sleight, and P. Suortti, J. Appl. Crystallogr. 23, 412 (1990).

${ }^{20}$ Y. Nakamura, G. Gebel, and P. Aldebert, J. Appl. Crystallogr. 22, 75 (1989).
${ }^{21}$ D. Orthaber, A. Bergmann, and O. Glatter, J. Appl. Crystallogr. 33, 218 (2000).

${ }^{22}$ A. Guinier and G. Fournet, Small Angle X-rays Scattering (Wiley, New York, 1955), p. 47

${ }^{23}$ L. Bosio, J. Teixeira, and H. E. Stanley, Phys. Rev. Lett. 46, 9 (1981)

${ }^{24}$ R. W. Hendricks, P. G. Mardon, and L. B. Shaffer, J. Chem. Phys. 61, 319 (1974).

${ }^{25}$ G. D. Wignall, J. Appl. Crystallogr. 24, 479 (1991).

${ }^{26}$ G. Porod, Z. Koll. 124, 83 (1951).

${ }^{27}$ Th. Zemb, in Neutron, X-Ray and Light Scattering: An Introduction to an Investigative Tool for Colloidal and Polymer Systems, edited by P. Lindner and Th. Zemb (North-Holland Delta Series, Amsterdam, 1991), pp. 177199.

${ }^{28}$ P. Lesieur, P. Lindner, C. Desforges, J. Lambard, and Th. Zemb, Physica B 180\&181, 564 (1992). 